\title{
Microstructure and pinning properties of hexagonal-disc shaped single crystalline $\mathrm{MgB}_{2}$
}

\author{
C. U. Jung, * J. Y. Kim, P. Chowdhury, Kijoon H. P. Kim, and Sung-Ik Lee ${ }^{\dagger}$ \\ National Creative Research Initiative Center for Superconductivity and Department of Physics, Pohang University of Science and \\ Technology, Pohang 790-784, Republic of Korea \\ D. S. Koh \\ Department of Physics, Pohang University of Science and Technology, Pohang 790-784, Republic of Korea
}

N. Tamura and W. A. Caldwell

Lawrence Berkeley National Laboratory, Advanced Light Source, 1 Cyclotron Road, MS-2-400 Berkeley, California 94720

J. R. Patel

Lawrence Berkeley National Laboratory, Advanced Light Source, 1 Cyclotron Road, MS 7-222 Berkeley, California 94720

and SSRL/SLAC, Stanford University, California 94309

(Received 6 March 2002; revised manuscript received 28 June 2002; published 27 November 2002)

\begin{abstract}
We synthesized hexagonal-disc-shaped $\mathrm{MgB}_{2}$ single crystals under high-pressure conditions and analyzed the microstructure and pinning properties. The lattice constants and the Laue pattern of the crystals from $\mathrm{x}$-ray micro-diffraction showed the crystal symmetry of $\mathrm{MgB}_{2}$. A thorough crystallographic mapping within a single crystal showed that the edge and $c$ axis of hexagonal-disc shape exactly matched the [10 $\overline{1} 0]$ and the [0001] directions of the $\mathrm{MgB}_{2}$ phase. Thus, these well-shaped single crystals may be the best candidates for studying the direction dependences of the physical properties. The magnetization curve and the magnetic hysteresis curve for these single crystals showed the existence of a wide reversible region and weak pinning properties, which supported our single crystals being very clean.
\end{abstract}

DOI: 10.1103/PhysRevB.66.184519

PACS number(s): 74.25.-q, 74.60.Ge, 74.72.- h

\section{INTRODUCTION}

The recent discovery ${ }^{1}$ of superconductivity in $\mathrm{MgB}_{2}$ has attracted great scientific ${ }^{2,3}$ and industrial ${ }^{4,5}$ interest. Even though basic issues such as the carrier type ${ }^{2}$ were addressed immediately, conflicting reports still exist, especially on the transport properties. ${ }^{6}$ For example, the residual resistivity ratio (RRR) of bulk $\mathrm{MgB}_{2}$ ranges from 1.2 to $30 .^{6}$

Higher quality bulk $\mathrm{MgB}_{2}$ (called high RRR-MgB ${ }_{2}$ ) has been claimed to have a higher values of RRR (20-25), a low residual resistivity $[\rho(40 \mathrm{~K})<1 \mu \Omega \mathrm{cm}]$, a higher magnetoresistance (MR), and a resistivity upturn at low temperature under high magnetic field. ${ }^{3}$ Insulating impurities and/or local strains have been suggested as possible origins for these different observations. ${ }^{7-11}$ However, very recently, the existence of unreacted $\mathrm{Mg}$ successfully explained the unusual enhancement of the RRR and the MR in polycrystalline $\mathrm{MgB}_{2} \cdot{ }^{6}$

Compared to the wide distribution of the RRR for polycrystals, the RRR in the $a b$-plane resistivity for single crystals has a narrow distribution. ${ }^{12,13}$ The RRR of single crystals ranges from 5 to 6 , which is much smaller than the values for high RRR-MgB ${ }_{2}$ bulk samples, but similar to the values for well-prepared polycrystals. ${ }^{6,14-16}$ The anisotropy factor $(\gamma$ $\sim 3)$ and the Debye temperature $(\Theta \sim 1160 \pm 60 \mathrm{~K})$ are also consistent among various reports. ${ }^{17}$

It is interesting to note that the magnetic properties are somewhat different for different single crystals. ${ }^{12,13,18}$ The superconducting transition width in the zero-field-cooled magnetization for single crystals as large as a few hundred $\mu \mathrm{m}$ is a little bit broader than expected. The ratio of the low-field magnetization in the field-cooled (FC) state to that in the zero-field-cooled (ZFC) state gives a rough indication of the pinning strength, and this ratio is quite different for different single crystals. The magnetic hysteresis for single crystals having very weak pinning can be used to probe impurities. In one report, the magnetic hysteresis contained a significant amount of paramagnetic component. ${ }^{13}$ In another report, the magnetic hysteresis for an aggregation of single crystals showed a large ferromagnetic contribution and a significant irreversible magnetization. ${ }^{18}$

For the above reasons, it is necessary to confirm the quality of single crystals in more detail before performing the main measurements. The existence of impurities and structural imperfections on a microscopic scale can result in diverse physical properties. Here, we report the growth of, as well as $\mathrm{x}$-ray microdiffraction and magnetization measurements for, $\mathrm{MgB}_{2}$ single crystals with hexagonal-disc shapes and shiny surfaces. Our single crystals are unique as far as the shape is concerned. The diagonal length and the thickness for the largest crystal was about 100 and $10 \mu \mathrm{m}$, respectively. The crystallinity was thoroughly identified by using the Laue pattern in the $\mathrm{x}$-ray microdiffraction measurement. Both the edge and the $c$ axis of the hexagonally shape disc were found to match the crystal symmetry. The magnetization study showed that pinning was very weak for our hexagonal-disc-shaped single crystals.

\section{EXPERIMENTS}

Two different procedures were used to grow the single crystals, and in both cases, excess $\mathrm{Mg}$ was critical for the growth of single crystals. The first involved a two-step 
method in which already synthesized pieces of $\mathrm{MgB}_{2}$ bulk $^{19,20}$ were used as a seed material. They were heat treated in a $\mathrm{Mg}$ flux inside a $\mathrm{Nb}$ tube, which was sealed in an inert gas atmosphere. Then, the $\mathrm{Nb}$ tube was put inside a quartz tube, which was sealed in vacuum. The quartz tube was heated for one hour at $1050{ }^{\circ} \mathrm{C}$, cooled very slowly to $700^{\circ} \mathrm{C}$ for five to fifteen days, and then quenched to room temperature. The temperature dependences of the $a b$-plane resistivities for $H \|_{a b}$ and $H \|_{c}$ and high-resolution transmission electron microscope images have already been reported for the single crystals synthesized using this procedure. ${ }^{21}$

In the one-step method, 1:1 mixtures of $\mathrm{Mg}$ and $\mathrm{B}$ powders were well ground and pressed into a pellet. ${ }^{12}$ Then, the pellet was placed in a Ta capsule. This capsule was put in a high-pressure cell equipped with a graphite heater. Heat treatment was done inside a 14-mm cubic multi-anvil-type press under $3 \mathrm{GPa} .{ }^{19,20}$ The heating temperature was around $1300^{\circ} \mathrm{C}$. The temperature was maintained for about $30 \mathrm{~min}-$ utes, and then was slowly lowered to $800-900{ }^{\circ} \mathrm{C}$. The final product was a pellet containing a mixture of singlecrystalline $\mathrm{MgB}_{2}$ and $\mathrm{Mg}$ flux.

It was found that the one-step method usually gave larger crystals, which was quite advantageous for the magnetization study in this research. The crystal images were observed using a polarizing optical microscope and a field-emission scanning electron microscope (SEM). We successfully separated single-crystalline $\mathrm{MgB}_{2}$ from the $\mathrm{Mg}$ flux by using a thermomechanical spinning method. This method is possible due to the fact that the melting (and/or decomposition) temperature of $\mathrm{MgB}_{2}$ is higher than that of $\mathrm{Mg}$.

Single crystals with sizes of tens of $\mu \mathrm{m}$ were selectively handled by using a homemade microtweezers and were fixed on Si substrates by using a photoresist as an epoxy. Since the volume of one crystal made in the one-step process was still rather small, we gathered about 200 single crystals on one substrate with their $c$ axes aligned perpendicular to the substrate surface. ${ }^{18}$ For the $\mathrm{x}$-ray micro-diffraction measurements, several crystals were fixed at the center of $\mathrm{Cu}$ crosshairs on the substrate, as shown in Fig. 1(d). ${ }^{21}$ The $\mathrm{Cu}$ crosshairs was used for the only purpose to facilitate the location of the sample by looking at the $\mathrm{Cu}$ fluorescence. The magnetic properties were measured with a SQUID magnetometer (Quantum Design, MPMS-XL).

The instrument used at the advanced light source (ALS) for $\mathrm{x}$-ray microdiffraction is capable of producing a submicron-size $\mathrm{x}$-ray microbeam and with submicron spatial resolution can probe the local texture in a single crystal. ${ }^{22}$ The sample was positioned using the $\mathrm{Cu}$ fluorescence signal detected from the $\mathrm{Cu}$ crosshairs on the $\mathrm{Si}$ substrate by using a high-purity Ge ORTEC solid-state detector connected to a multichannel analyzer. The crystal orientation with respect to the substrate can be determined with an accuracy of $0.01^{\circ}$.

\section{RESULTS AND DISCUSSION}

\section{A. SEM and microXRD}

Figure 1(a) shows a typical SEM image for a $\mathrm{MgB}_{2}$ single crystal synthesized in a two-step process. The crystals ob-

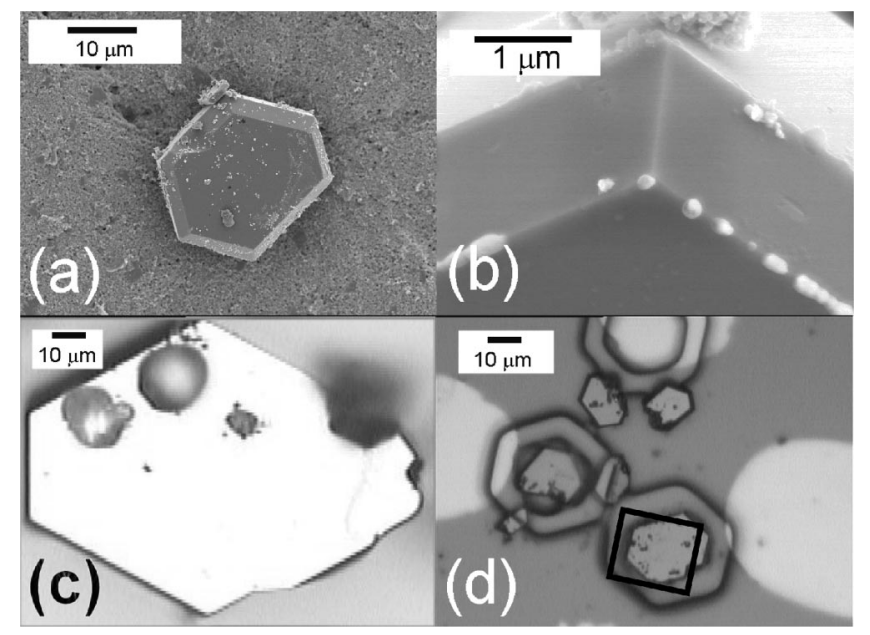

FIG. 1. SEM and polarizing optical microscope images of the $\mathrm{MgB}_{2}$ single crystals with a hexagonal-disc shape. (a) $\mathrm{MgB}_{2}$ made in a two-step process with a diagonal distance of about $25 \mu \mathrm{m}$ and with a thickness of about $4 \mu \mathrm{m}$. (b) High-resolution image at the corner of another crystal, and (c) polarizing optical microscope image of a single crystal grown in a one-step process. (d) Polarizing optical microscope images of $\mathrm{MgB}_{2}$ single crystals. An epoxy was used to fix six single crystals at the center of $100-\mu$ m-wide $\mathrm{Cu}$ crosshairs. The crystal enclosed by a rectangle was scanned for microstructural mapping in Fig. 2.

served by using a polarizing optical microscope had hexagonal-disc shapes with edge angles of $120^{\circ}$ and very flat and shiny surfaces. The sizes of the crystals were about $20-60 \mu \mathrm{m}$ in diagonal length and $2-6 \mu \mathrm{m}$ in thickness. Figure 1(b) shows a magnified view of the upper corner of another crystal. The smooth surfaces and the sharp edges confirm that our small crystals had a very low probability of having mosaic aggregates of nanocrystals either along the $a b$ plane or along the $c$ axis; thus, we had a better chance to study their intrinsic properties. The single crystals reported so far, except for those reported by us, have had irregular shapes. ${ }^{12,13,18}$

Figure 1(c) shows a polarizing optical microscope image of a $\mathrm{MgB}_{2}$ single crystal synthesized in a one-step process, which resulted in larger crystals. The sizes of these crystals were $30-100 \mu \mathrm{m}$ in diagonal length, so they were picked for measurements of the magnetic properties and the x-ray microdiffraction.

The crystal structure was identified by using white beam $\mathrm{x}$-ray microdiffraction measurements. After positioning these single crystals on the substrate in Fig. 1(d), a $100 \mu \mathrm{m}$ $\times 100 \mu \mathrm{m}$ region between the $\mathrm{Cu}$ crosshairs was scanned with a step size of $2 \mu \mathrm{m}$. At each step, the Laue pattern (together with the $\mathrm{Cu} K$ fluorescence signal) was collected with a BRUKER 6000 CCD camera which has an active area of $9 \times 9 \mathrm{~cm}$ and was placed about $4 \mathrm{~cm}$ above the sample (2500 images, 1024 pixel $\times 1024$ pixel mode). The exposure time at each step was $1 \mathrm{~s}$. An example of a Laue pattern obtained from a $\mathrm{MgB}_{2}$ single crystal is shown in Fig. 2(a) (for clarity, the color of screen was changed from blue to black). The Laue patterns with refined lattice parameters of $a=3.0867 \pm 0.0003 \AA, c=3.5235 \pm 0.0003 \AA$ showed excel- 


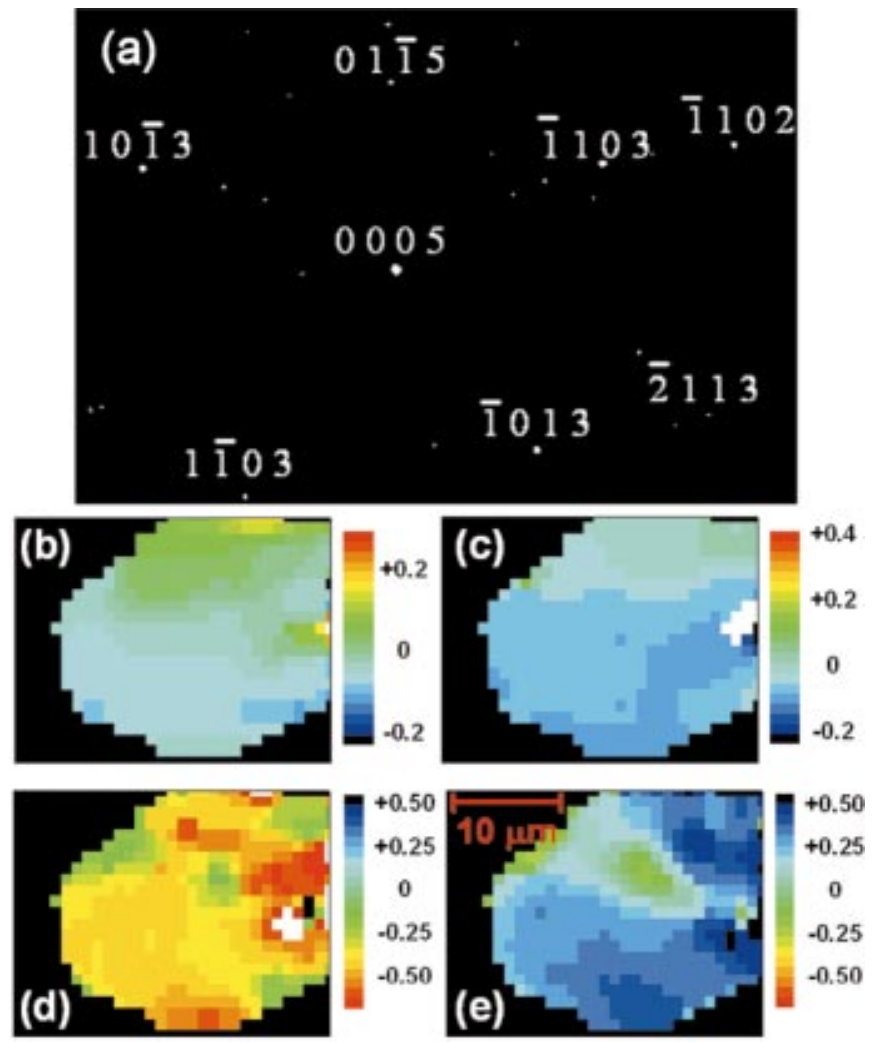

FIG. 2. (Color) The region enclosed by the rectangle in Fig. 1(d) was scanned with $1 \mu$ grid and the scanned value at each spot was denoted by a colored pixel with $1 \mu \mathrm{m} \times 1 \mu \mathrm{m}$ area. (a) A representative image of an indexed Laue pattern from xray microdiffraction. (b) and (c) are, respectively, for the out-of-plane and the in-plane orientations inside a single crystal. Note that difference from mean values $\left(6.0^{\circ}\right.$ and $26.0^{\circ}$, respectively) is shown in the vertical color bars. The strains for the [1010] and [0001] directions are shown in (d) and (e) where vertical color scale are in units of $10^{-3}$ in strain. The lateral scales are the same for (b), (c), (d), and (e) and a scale bar of $10 \mu \mathrm{m}$ is only shown in (e). Outer black regions in (b), (c), (d), and (e) correspond to the Si substrate and black or white pixels inside the crystal area correspond to the defects explained in the text.

lent agreement with a hexagonal $\mathrm{MgB}_{2}$ structure ( $a$ $=3.086 \AA$, $c=3.524 \AA$, Space Group number $=191$, Ref. $1)$. The silicon reflections from the substrate could be digitally removed. Typically, more than 20 reflections with energies ranging from 5 to $14 \mathrm{keV}$ were indexed successfully. [For clarity, only brighter spots were indexed in the Fig. 2(a).] The (0005) reflection in the center of the pattern in Fig. 2 corresponds to the direction of the normal to the crystal surface. This confirms that the surface plane normal is along the $c$ axis. Moreover, the hexagonal edges of the crystals were found to match the [10 10$]$ directions within a fraction of a degree resolution. Thus, the shapes of the crystals in the microscope image followed the $\mathrm{MgB}_{2}$ crystal symmetry, which will be quite useful for any research of the direction dependencies of the physical properties in $\mathrm{MgB}_{2}$.

Indexing the Laue patterns in Fig. 2(a) allowed us to calculate the complete orientation matrix of the x-ray illuminated volume. A finer step size of $1 \mu \mathrm{m}$ was used for the white-beam scan. The orientation variations inside the single crystal shown in the right bottom corner of Fig. 1(d) are shown in Figs. 2(b) and 2(c). Here the deviations of angle from the mean values $\left[6.0^{\circ}\right.$ and $26.0^{\circ}$, respectively $]$ are represented by a colored pixel with $1 \mu \mathrm{m} \times 1 \mu \mathrm{m}$ area around the measured spot. Figure 2(b) is the out-of-plane orientation variation calculated as the angle between the $c$ axis and the normal to the surface of the silicon substrate. The gradual change of color from top to bottom of the crystal means that angle between the crystal $c$ axis and substrate $c$ axis changes from 6.2 to 5.8, indicating a slight bending of the crystal. This assumption is also compatible with the fact that change of angle along the horizontal direction in the figure is much smaller. Another thing is that the crystal was put declined by 6 degrees over Si substrate, which can result in bending of thin crystal during curing of epoxy existing between the crystal and the substrate. Thus, the real $c$ axis distribution seems to be quit lower than observed.

Figure 2(c) shows the in-plane orientation variation calculated as the angle between the measured [10 10$]$ direction and a reference direction (horizontal direction in the figure). The gradual change of color from top to bottom means that the angle changes from 26.2 to 25.8 . The average value of 26.0 is just the same as that of the angle between one of the crystal edges and the reference direction. The in-plane orientation also showed some inhomogeneities of up to about $0.2^{\circ}$. Some small region colored with white or black showed a larger deviation of axes from other main region and might be due to some defects.

These mapping of orientation demonstrate that the orientation of crystal axis of our hexagonal-disc-shaped single crystals was perfect, within $0.2^{\circ}$. A recent study showed that (0001) twist grain-boundaries, formed by rotations along the $c$ axis (typically by about $4^{\circ}$ ), were the major grain boundaries in polycrystalline $\mathrm{MgB}_{2}{ }^{7}$ This kind of grain boundary was attributed to the weaker bonding between $\mathrm{Mg}$ layer and B layer. ${ }^{7}$ Note that bending observed in Fig. 2(b) could not give significant overestimation of in-plane axis distribution, the real $c$-axis distribution seems to be lower than observed and lower than in-plane axis distribution of the single crystal. This is also compatible with weak bonding between $\mathrm{Mg}$ layer and B layer.

The local strain mapping may also give information on the microstructure of the single crystal. The deviations of the reflections positions from unstrained crystal lattice yields the deviatoric (distortional) components of the strain (distortion of the unit cell). Or, without reference crystal lattice, it can gives a strain distribution. Figures 2(d) and 2(e) show the deviatoric component along [10 $\overline{1} 0]$ and [0001], respectively. The color scale are in units of $10^{-3}$ in strain. All the distortions are calculated relatively to the lattice constant values given in Nagamatsu's paper. ${ }^{1}$

For both orientation, the regions with low strain are commonly observed near the center and peripheral region, which supports quantitative analysis as well as some qualitative analysis. ${ }^{23}$ Compared with polycrystal, the single crystal appears to be weakly biaxially strained in tension as indicated by the dominant positive value (red) for the in-plane unit cell axes compared to the dominant negative (blue) value for the 
out-of-plane axis . The overall strain variations of about 6 $\times 10^{-4}$ across the crystal surface is quite low but higher than the technique resolution of about $10^{-4}$ and would correspond in variation of an unit on the last digit of Akimitsu's lattice parameter. The average strain values were $\epsilon_{11}=0.231 \times(1$ $\pm 0.141) 10^{-3}$ and $\epsilon_{33}=-0.481 \times(1 \pm 0.154) 10^{-3}$. As in the case of the orientation map in Fig. 2, we observed some local variations of the strain departing from these average values, indicated by the white or black colored spots in the strain maps. The reproducibility of these data was checked by performing two separate scans of the same area.

The influence of lattice strain on the superconducting properties has been investigated also for polycrytalline $\mathrm{MgB}_{2} .{ }^{11}$ The variations in lattice strain and $\mathrm{Mg}$ vacancy concentrations were obtained by varying the synthesis conditions. It was found that high strain $(\sim 1 \%)$ and the presence of $\mathrm{Mg}$ vacancies $(\sim 5 \%)$ resulted in lowering the $T_{c}$ by only $2 \mathrm{~K}$. Thus the strain of our crystals was more than 3 orders of magnitude smaller than that of polycrystals, which implies the strain or $\mathrm{Mg}$ vacancy may not account for the $T_{c}$ difference between our single crystal and polycrystals.

\section{B. Magnetic properties}

To study the bulk nature of the superconductivity, we measured the magnetization curve $M(T)$ and the magnetic hysteresis $M(H)$. Since the volume of one crystal was rather small, we fixed about 30 single crystals made in the one-step process on a substrate without appreciable magnetic background with their $c$ axes aligned perpendicular to the substrate surface. ${ }^{21}$ Figure 3 (a) shows the magnetization curve $M(T)$ measured at $20 \mathrm{Oe}$ in the ZFC and the FC states. The $T_{c}$ onset was about $38 \mathrm{~K}$. The FC signals for fields parallel and perpendicular to the $c$ axis were larger than $60 \%$ of the ZFC signals, which suggested that the pinning was very weak. The overall shape was not much different for these two orientations. The different values of the magnetic moment for the different field directions were due to a demagnetization effect caused by the planar-disc shapes of the crystals. ${ }^{21}$ The transition width in the $M(T)$ curve was much narrower than that for single crystals made in the two-step method. ${ }^{21}$ We also found that the average volume of the crystals made in the one-step process were about 25 times larger than the average volume of the crystals made in the two-step process in the previous study ${ }^{21}$ by the comparison of the ZFC signal per one single crystal.

The weak pinning was demonstrated by the magnetization at $5000 \mathrm{Oe}$ and the magnetic hysteresis $M(H)$ at $5 \mathrm{~K}$, as shown in Figs. 3(b) and 3(c), respectively. With $\left[M\left(H^{-}\right)\right.$ $\left.-M\left(H^{+}\right)\right]=0.1 \mathrm{emu} / \mathrm{cm}^{3}$ as the criterion for the reversible point, the values of $T_{\text {irr }}(H=5000 \mathrm{Oe})$ were 26 and $24 \mathrm{~K}$ for $H \| c$ and $H \| a b$, respectively. At each temperature, the upper critical fields obtained from the resistivity measurements were about 1.7 and $6 \mathrm{~T}$ for our well-shaped single crystals. ${ }^{21}$ Thus, a very wide reversible region existed in our single crystals, especially for $H \| a b$. ( $H_{\text {irr }}$ for bulk $\mathrm{MgB}_{2}$ at $T$ $=25 \mathrm{~K}$ was about $3 \mathrm{~T},{ }^{16}$ which is about 6 times higher than that of single crystalline $\mathrm{MgB}_{2}$.)
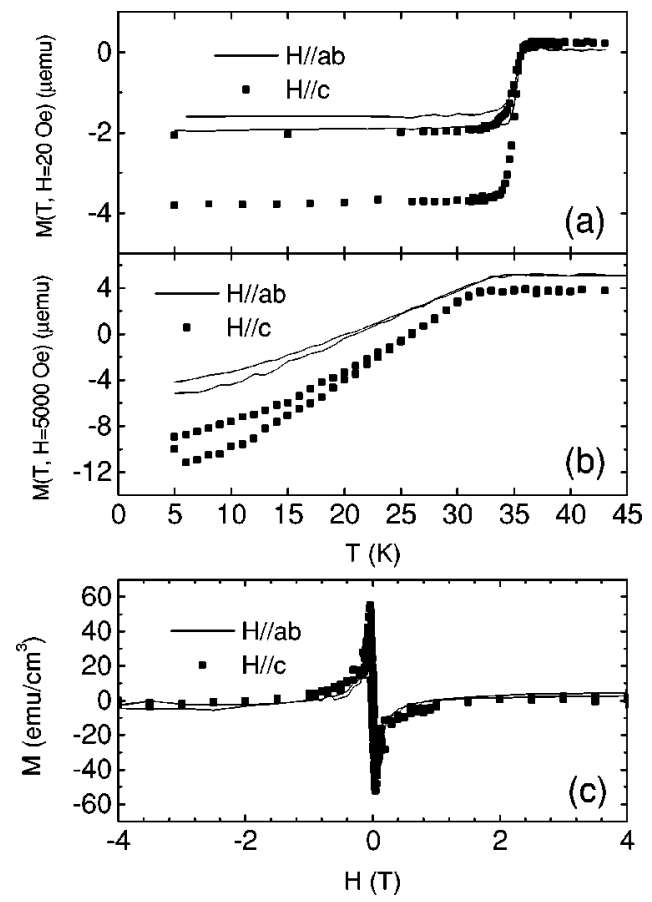

FIG. 3. (a) Low-field magnetization curve $M(T)$ measured at 20 Oe for $H \| c$ and $H \| a b$. (b) Magnetization curves measured at 5000 Oe showed a wide reversible region. $T_{\text {irr }}(H=5000 \mathrm{Oe})$ was 26 and $24 \mathrm{~K}$ for $H \|_{c}$ and $H \| a b$, respectively. (c) The magnetic hysteresis $M(H)$ at $5 \mathrm{~K}$; the symbols and the line are for $H \|_{c}$ and $H \| a b$, respectively.

The weak pinning shown by the wide reversible region was also consistent with the results of the $M(H)$ measurement. The $M(H)$ at $5 \mathrm{~K}$ in Fig. 3(c) showed negligible paramagnetic or ferromagnetic background up to $5 \mathrm{~T}$. Reversible magnetization was dominant for the $M(H)$ of our single crystals, which was quite consistent with fact that the pinning in our single crystals was weak and with the existence of wide reversible region demonstrated by the lower $H_{\text {irr }}$ value in Fig. 3(b). The different slopes of the $M(H)$ curves at the starting low fields $(H<300 \mathrm{Oe})$ for the different field directions were due to different demagnetization factors.

Previously, the $M(H)$ for irregular shaped single crystals was shown to have a non-negligible irreversible contribution $^{18}$ and a quite large paramagnetic and/or ferromagnetic background signal, which might have been due to the existence of impurities. ${ }^{13,18}$ The results in Fig. 3 indicate that the strong bulk pinning previously reported for polycrystalline $^{24}$ and thin films ${ }^{4}$ might be due to entirely extrinsic pinning sites, such as grain boundaries and crystallographic defects. ${ }^{25}$ This is consistent with the absence of core pinning, even at $T \sim 0.5 \times T_{c}$, for bulk sample. ${ }^{5}$

\section{SUMMARY}

In summary, we report the structural and magnetic properties of $\mathrm{MgB}_{2}$ single crystals with hexagonal-disc shapes. The x-ray microdiffraction showed that the hexagonal-disc shape of the single crystal followed the crystallographic symmetry, which will be very useful for studying 
orientation-dependent physical properties. Detailed crystallographic mapping was somewhat consistent with the crystal structure with weak bonding between $\mathrm{Mg}$ layer and B layer. The magnetization curve and the magnetic hysteresis provided consistent evidence that our single crystals were very clean and had very weak pinning: the large FC magnetization and the sharp transition of the ZFC magnetization at low field $M(T)$, the wide reversible region, and the $M(H, T$ $=5 \mathrm{~K})$ dominated by the reversible magnetization without any significant paramagnetic and/or ferromagnetic contribution.

\section{ACKNOWLEDGMENTS}

We appreciate M. H. Kim and S. R. Jung for their help in handling of tiny crystals. This work is supported by the Ministry of Science and Technology of Korea through the Creative Research Initiative Program. The Advanced Light Source is supported by the Director, Office of Science, Office of Basic Energy Sciences, Materials Sciences Division, of the U.S. Department of Energy under Contract No. DEAC03-76SF00098 at Lawrence Berkeley National Laboratory.
*Present address: Tokura Spin Superstructure Project, ERATO, JST, AIST, Tsukuba Central 4 Tsukuba, Ibaraki 305-8562, Japan. Email address: cu-jung@aist.go.jp. URL: http://unit.aist.go.jp/ cerc/

†Email address: silee@ postech.ac.kr. URL: http://wwwpsc.postech.ac.kr

${ }^{1}$ J. Nagamatsu, N. Nakagawa, T. Muranaka, Y. Zenitani, and J. Akimitsu, Nature (London) 410, 63 (2001).

${ }^{2}$ W. N. Kang, C. U. Jung, Kijoon H. P. Kim, Min-Seok Park, S. Y. Lee, Hyeong-Jin Kim, Eun-Mi Choi, Kyung Hee Kim, MunSeog Kim, and Sung-Ik Lee, Appl. Phys. Lett. 79, 982 (2001).

${ }^{3}$ S. L. Bud'ko, C. Petrovic, G. Lapertot, C. E. Cunningham, P. C. Canfield, M-H. Jung, and A. H. Lacerda, Phys. Rev. B 63, 220503 (2001); D. K. Finnemore, J. E. Ostenson, S. L. Bud'ko, G. Lapertot, and P. C. Canfield, Phys. Rev. Lett. 86, 2420 (2001).

${ }^{4}$ W. N. Kang, Hyeong-Jin Kim, Eun-Mi Choi, C. U. Jung, and Sung-Ik Lee, Science 292, 1521 (2001).

${ }^{5}$ D. C. Larbalestier, L. D. Cooley, M. O. Rikel, A. A. Polyanskii, J. Jiang, S. Patnaik, X. Y. Cai, D. M. Feldmann, A. Gurevich, A. A. Squitieri, M. T. Naus, C. B. Eom, E. E. Hellstrom, R. J. Cava, K. A. Regan, N. Rogado, M. A. Hayward, T. He, J. S. Slusky, P. Khalifah, K. Inumaru, and M. Haas, Nature (London) 410, 186 (2001)

${ }^{6}$ C. U. Jung, Heon-Jung Kim, Min-Seok Park, Mun-Seog Kim, J. Y. Kim, Zhonglian Du, Sung-Ik Lee, K. H. Kim, J. B. Betts, M. Jaime, A. H. Lacerda, and G. S. Boebinger, Physica C 377, 21 (2002); K. H. Kim, J. B. Betts, M. Jaime, A. H. Lacerda, G. S. Boebinger, C. U. Jung, Heon-Jung Kim, Min-Seok Park, and Sung-Ik Lee, Phys. Rev. B 66, 020506 (2002).

${ }^{7}$ Y. Zhu, L. Wu, V. Volkov, Q. Li, G. Gu, A. R. Moodenbaugh, M. Malac, M. Suenaga, and J. Tranquada, Physica C 356, 239 (2001).

${ }^{8}$ X. H. Chen, Y. S. Wang, Y. Y. Xue, R. L. Meng, Y. Q. Wang, and C. W. Chu, Phys. Rev. B 65, 024502 (2002).

${ }^{9}$ Y. Y. Xue, R. L. Meng, B. Lorenz, J. K. Meen, Y. Y. Sun, and C. W. Chu, Physica C 377, 7 (2002).

${ }^{10}$ B. Lorenz, Y. Y. Xue, R. L. Meng, and C. W. Chu, cond-mat/0110125 (unpublished).

${ }^{11}$ A. Serquis, Y. T. Zhu, E. J. Peterson, J. Y. Coulter, D. E. Peterson, and F. M. Mueller, Appl. Phys. Lett. 79, 4399 (2001).

${ }^{12}$ S. Lee, H. Mori, T. Masui, Yu. Eltsev, A. Yamamoto, and S.
Tajima, J. Phys. Soc. Jpn. 70, 2255 (2001).

${ }^{13}$ M. Xu, H. Kitazawa, Y. Takano, J. Ye, K. Nishida, H. Abe, A. Matsushita, N. Tsujii, and G. Kido, Appl. Phys. Lett. 79, 2779 (2001); A. K. Pradhan, Z. X. Shi, M. Tokunaga, T. Tamegai, Y. Takano, K. Togano, H. Kito, and H. Ihara, Phys. Rev. B 64, 212509 (2001).

${ }^{14}$ N. A. Frederick, S. Li, M. B. Maple, V. F. Nesterenko, and S. S. Indrakanti, Physica C 363, 1 (2001).

${ }^{15}$ S. S. Indrakanti, V. F. Nesterenko, M. B. Maple, N. A. Frederick, W. M. Yuhasz, and Shi Li, Philos. Mag. Lett. 81, 849 (2001).

${ }^{16}$ G. Fuchs, K. -H. Müller, A. Handstein, K. Nenkov, V. N. Narozhnyi, D. Eckert, M. Wolf, and L. Schultz, Solid State Commun. 118, 497 (2001).

${ }^{17}$ However, even in single crystals, some spectra for the transport properties still exist. MR values at $5 \mathrm{~T}$ ranged from about 2 to $20 \%$ and the MR ratio for the two magnetic field directions shows a wide variation among reports.

${ }^{18}$ O. F. de Lima, R. A. Ribeiro, M. A. Avila, C. A. Cardoso, and A. A. Coelho, Phys. Rev. Lett. 86, 5974 (2001).

${ }^{19}$ C. U. Jung, Min-Seok Park, W. N. Kang, Mun-Seog Kim, Kijoon H. P. Kim, S. Y. Lee, and Sung-Ik Lee, Appl. Phys. Lett. 78, 4157 (2001).

${ }^{20}$ C. U. Jung, J. Y. Kim, Min-Seok Park, Heon-Jung Kim, MunSeog Kim, and Sung-Ik Lee, Phys. Rev. B 65, 172501 (2002).

${ }^{21}$ Kijoon H. P. Kim, Jae-Hyuk Choi, C. U. Jung, P. Chowdhury, Hyun-Sook Lee, Min-Seok Park, Heon-Jung Kim, J. Y. Kim, Zhonglian Du, Eun-Mi Choi, Mun-Seog Kim, W. N. Kang, Sung-Ik Lee, Gun Yong Sung, and Jeong Yong Lee, Phys. Rev. B 65, 100510 (2002).

${ }^{22}$ A. A. MacDowell, R. S. Celestre, N. Tamura, R. Spolenak, B. C. Valek, W. L. Brown, J. C. Bravman, H. A. Padmore, B. W. Batterman, and J. R. Patel, Nucl. Instrum. Methods Phys. Res. A 467-468, 936 (2001).

${ }^{23}$ This coincidence removes a lot the possible artifacts due to different effective numbers of lattice constants from our single crystal and polycrystal. These values are only $0.04 \%$ different with each other.

${ }^{24}$ Mun-Seog Kim, C. U. Jung, Min-Seok Park, S. Y. Lee, Kijoon H. P. Kim, W. N. Kang, and Sung-Ik Lee, Phys. Rev. B 64, 012511 (2001).

${ }^{25}$ M. Pissas, E. Moraitakis, D. Stamopoulos, G. Papavassiliou, V. Psycharis, and S. Koutandos, J. Supercond. 14, 615 (2001). 